Algebraic $\&$ Geometric $\mathcal{T}_{\text {opology }}$

Volume 5 (2005) 509-535

Published: 4 June 2005

ATG

\title{
Minimal surface representations of virtual knots and links
}

\author{
H.A. DYe \\ Louis H. KaufFman
}

\begin{abstract}
Kuperberg [15] has shown that a virtual knot diagram corresponds (up to generalized Reidemeister moves) to a unique embedding in a thickened surface of minimal genus. If a virtual knot diagram is equivalent to a classical knot diagram then this minimal surface is a sphere. Using this result and a generalised bracket polynomial, we develop methods that may determine whether a virtual knot diagram is non-classical (and hence non-trivial). As examples we show that, except for special cases, link diagrams with a single virtualization and link diagrams with a single virtual crossing are non-classical.
\end{abstract}

AMS Classification 57M25, 57M27; 57N05

Keywords Virtual knots, minimal surface representation, bracket polynomial, Kishino knot

\section{Introduction}

Virtual knot diagrams are a generalization of classical knot diagrams introduced by L. Kauffman in 1996 [9]. Results in this area immediately indicated that the bracket polynomial and the fundamental group did not detect many nontrivial and non-classical virtual knot diagrams. We are interested in detecting non-trivial virtual knot diagrams, and, in particular, determining if a virtual knot diagram is non-classical and non-trivial.

The bracket polynomial and the fundamental group can not differentiate all nontrivial virtual knot diagrams from the unknot. Kauffman, in [9], gave a process of virtualization that produces from a diagram $K$, a pair of diagrams: a virtual knot diagram $K_{v}$ and a classical knot diagram $K_{s}$ (obtained by switching a crossing in $K$ ). The diagrams $K_{v}$ and $K_{s}$ have the same bracket polynomial. One can show that if $K$ is a non-trivial classical knot, then $K_{v}$ is a non-trivial virtual knot (possibly classical) 9]. This process may be used to construct 
non-trivial virtual knot diagrams with trivial bracket polynomial. There are also other virtual knot diagrams with trivial bracket polynomial that are not produced by virtualization. Kishino's knot is the first example of this type and it is not differentiated from the unknot by the fundamental group or the Jones polynomial. In [13, Kishino's knot was detected by the 3 -strand bracket polynomial. Kishino's knot is also detected by the quaternionic biquandle [2], [11. However, these invariants can be difficult to compute. Other virtual knot diagrams, undetected by the fundamental group and the bracket polynomial, are described in [4. It is shown in [4] that there are an infinite number of virtual knot diagrams that are not detected by the fundamental group or the Jones polynomial.

Using the bracket polynomial and Kuperberg's result [15, we develop methods that may determine if a virtual knot diagram is non-trivial and non-classical. We focus on the case of knot diagrams with one virtualization and the examples in [4. We show that, except for special cases, link diagrams with a single virtualization and link diagrams with a single virtual crossing are non-classical and non-trivial. We construct examples of virtual link diagrams with either one virtualization or one virtual crossing using the methods from [19] that are not detectable by the surface bracket polynomial. In the final section, we discuss virtual knots produced by two virtualizations.

\section{Virtual knots and links and minimal representa- tions}

Virtual knot (and link) theory was introduced by the second author in 9], to which we refer for basic concepts and notation. In particular we use a small circle to indicate a virtual crossing in a diagram. A virtual knot (or link) is an equivalence class of diagrams containing ordinary crossings and virtual crossings under the three familar Reidemeister moves together with the ability to move an arc containing only virtual crossings to any other position with the same endpoints. A virtual link diagram can be represented as a link diagram in an oriented surface, by adding a handles to desingularize the virtual crossings. This can also be done by regarding the diagram as lying in a surface with boundary (cf Kamada and Kamada [7]) and capping the boundary components. This surface can be stabilized by adding further handles which do not meet the diagram. This diagram can then be regarded as a genuine link in the surface thickened by crossing with a unit interval. Carter, Kamada and Saito [3] have shown that desingularization induces a bijection between equivalence classes of 
virtual links and stable equivalence classes of links in a thickened surface. A short proof of this result and a good summary of the various different ways of thinking of a virtual link can be found in [5, Theorem 4.5 and above], see also 8, 12. In 12 the Kuperberg result (below) is used to show that virtual knots are algorithmically recognizable and to find the genus of connected sums of virtual knots.

A representation of a surface is minimal if it cannot be destabilized.

Theorem 2.1 (Kuperberg [15]) A minimal representation of a virtual link is unique up to homeomorphism of the thickened surface.

Corollary 2.2 If the minimal surface has genus greater than zero then the link is non-trivial and not equivalent to a classical link.

We need to think of these results in terms of diagrams, rather than thickened surfaces. In these terms destabilization and is performed by surgering the surface along a cancellation curve which does not meet the diagram. A representation of a link is then minimal if no cancellation curve can be found after a possible sequence of Reidemeister moves. We refer to a minimal representation as a characterization and we define the virtual genus of a virtual knot or link diagram to be the minimal genus.

Corollary 2.2 taken alone does not provide an algorithm that determines cancellation curves. However, such algorithms can be formulated using normal surface theory 16, 18. In this paper we use algebraic invariants of knots and links to investigate minimality.

We need to stress the non-constructive nature of minimality:

Remark To obtain a characterization from a representation it may be necessary to perform a sequence of handle cancellations and Reidemeister moves combined with non-trival homeomorphisms of the surfaces.

We will introduce new methods which apply a generalization of the bracket polynomial to representations of virtual knot diagrams. These methods often determine if a given representation is minimal and hence, by Corollary 2.2 . if a virtual knot diagram is non-classical and non-trivial. One method uses homology classes and intersection numbers to determine non-triviality and the other method uses isotopy classes to determine non-triviality. Both methods utilize the bracket skein relation to produce states that consist of simple closed 
curves in the surface with coefficients in $\mathbb{Z}\left[A, A^{-1}\right]$ from a fixed representation. We make the following definitions.

For a fixed representation of a virtual knot diagram, we refer to the surface-knot pair, $(F, K)$, to indicate a specific choice of surface and embedding of the knot. A surface-state pair, $(F, s)$, is a collection of disjoint simple closed curves in the surface.

We obtain a surface-state pair $(F, s)$ from $(F, K)$ by assigning a smoothing type to each classical crossing in the surface.

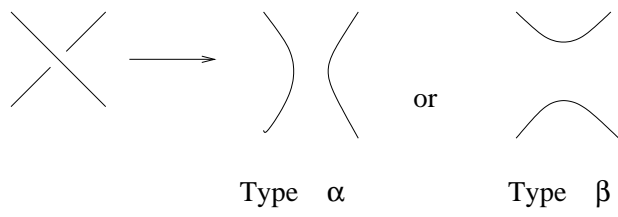

Figure 1: Smoothing Types

Note that if $(F, K)$ has $n$ classical crossings we obtain $2^{n}$ surface-state pairs, denoted $\left\{\left(F, s_{1}\right), \ldots\left(F, s_{2^{n}}\right)\right\}$, by assignment of smoothing type. We denote the collection of all surface-state pairs as $(F, S)$. A surface state pair is analogous to a state of a classical knot diagram.

We consider a surface-state pair, $(F, s)$, in more detail. Each surface-state pair is a collection of disjoint simple closed curves on the surface $F$. For a fixed collection of disjoint sets of curves in the surface $\mathrm{F}$ we may study either isotopy classes of these curves (with or without homeomorphisms) or homology classes of curves.

We define the surface bracket polynomial of a representation $(F, K)$. Let $\hat{K}$ be a virtual knot diagram and let $(F, K)$ be a fixed representation of $\hat{K}$. The surface bracket polynomial of $K$ is denoted as $\langle(F, K)\rangle$. Then:

$$
\langle(F, K)\rangle=\sum_{(F, s(c)) \in(F, S)}\langle K \mid s(c)\rangle d^{|s(c)|}[s(c)]
$$

where $\langle K \mid s(c)\rangle=A^{c(s)}$ and $c(s)$ is the number of type $\alpha$ smoothings minus the number of type $\beta$ smoothings. $|s(c)|$ is the number of curves which bound a disk in the surface and $[s(c)]$ represents a formal sum of the disjoint curves that do not bound a disk in the surface-state pair $(F, s(c))$.

(Note that $[s(c)]$ may be regarded either as a formal sum of homology classes in the surface $\mathrm{F}$ or as a sum of isotopy classes in the surface $\mathrm{F}$ mod orientation preserving homeomorphisms of F.) We may also compute the surface bracket 
polynomial by applying the skein relation with the axiom: $\langle F, U\rangle=d=-A^{2}-$ $A^{-2}$ if $U$ bounds a disk in $F$. In [17, Manturov introduces related polynomial invariants of knots in 2-surfaces. The surface bracket polynomial is invariant under the Reidemeister moves II and III.

Remark After collecting coefficients of equivalent (homologous or isotopic) surface-state pairs there may be fewer than $2^{n}$ surface-state pairs with nonzero coefficients.

We focus on using the homology classes of the curves in the surface-state pairs to determine if a virtual knot diagram is non-trivial. Since $F$ is a closed, orientable surface, we can write $F=T_{1} \sharp T_{2} \ldots \sharp T_{n}$ where each $T_{i}$ is a torus. The homology group, $H_{1}(F)$, is generated by $\left\{\left[m_{1}\right] \ldots\left[m_{n}\right],\left[l_{1}\right] \ldots\left[l_{n}\right]\right\}$ where $\left[m_{i}\right]$ and $\left[l_{i}\right]$ represent the homology class of the meridian and longitude of the torus $T_{i}$ respectively. If $\gamma$ is a curve in the surface-state pair $(F, s)$ then either $\gamma$ is homologically trivial in $F$ or $\gamma$ is homologous to a curve of the form:

$$
\sum_{i=1 \ldots n} a_{i}\left[m_{i}\right]+b_{i}\left[l_{i}\right]
$$

where $a_{i}$ and $b_{i}$ are relatively prime.

The relationship between a cancellation curve, surface-knot pair and surfacestate pairs is expressed in the following lemma.

Lemma 2.3 Let $C$ be a cancellation curve for a representation, $(F, K)$, of a virtual knot diagram, $\hat{K}$. Then $C$ is a cancellation curve for every surface-state pair.

Proof Suppose that $C$ is a cancellation curve for $(F, K)$, but $C$ is not a cancellation curve for some surface-state pair $(F, s)$ obtained from the $(F, K)$. This indicates that either $C$ intersects a curve in the surface-state pair $(F, s)$ or $C$ bounds a disk in the surface $F$. We assumed that $C$ was a cancellation curve for the representation, so $C$ does not bound a disk in $F$. If the curve $C$ intersects the state $s$ then then the curve $C$ also intersects the original diagram $K$ as a result of the definition in Figure 1. Hence, $C$ is not a cancellation curve for this representation $(F, K)$, contradicting our original assumption.

We define the intersection number of two oriented curves, $\alpha$ and $\beta$, in a surface $F$ to be the intersection number between the elements $[\alpha]$ and $[\beta]$ of the homology classes $H_{1}(F, \mathbb{Z})$. We will denote this as $[\alpha] \bullet[\beta]$. Recall from [1 that intersection number is the Poincare dual to the cup product, and that it can be 
calculated by placing the two curves transversely to each other and counting the sum of the oriented intersections of them.

In the next theorem we give conditions for the existence of a cancellation curve $C$ for a representation $(F, K)$ of a virtual knot diagram $\hat{K}$. The conditions are given in terms of intersection numbers of the corresponding surface state pairs with generators of homology of the surface. By applying these conditions to the surface state pairs having non-zero coefficients in the surface bracket polynomial, we find conditions for the existence of cancellation curves for all diagrams obtained from $(F, K)$ by isotopy and Reidemeister moves, in other words conditions for the minimality of $(F, K)$.

Theorem 2.4 Let $(F, K)$ be a representation of a virtual knot diagram with $F=T_{1} \sharp T_{2} \ldots \sharp T_{n}$. Let

$$
\left\{\left(F, s_{1}\right),\left(F, s_{2}\right) \ldots\left(F, s_{m}\right)\right\}
$$

denote the collection of surface-state pairs obtained from $(F, K)$. Assign an arbitrary orientation to each curve in the surface-state pairs. Let $p: F \rightarrow T_{k}$ be the collapsing map, and let $p_{*}: H_{1}(F, \mathbb{Z}) \rightarrow H_{1}\left(T_{k}, \mathbb{Z}\right)$ be the induced map on homology. If for each $T_{k}$ there exist two states $s_{i}$ and $s_{j}$ with non-zero coefficients that contain curves (with arbitrarily assigned orientation) $\gamma_{i}$ and $\gamma_{j}$ respectively, such that $p_{*}\left[\gamma_{i}\right] \bullet p_{*}\left[\gamma_{j}\right] \neq 0$ then there is no cancellation curve for $(F, K)$.

Proof We initially assume that $F$ is a torus, $T$. Note that $p: F \rightarrow T$ is the identity map in this case. Suppose $(T, K)$ has a cancellation curve $C$. Let $s_{i}$ and $s_{j}$ be two states with non-zero coefficients that contain curves with nonzero intersection number in the torus $T$. The curve $C$ is a cancellation curve and therefore does not intersect any curve in the two states, $s_{i}$ or $s_{j}$. In the torus, each state consists of a collection of curves that are parallel copies of a simple closed curve with non-trivial homology class in $H_{1}(T, \mathbb{Z})$ and curves that bound a disk in the surface $T$ after homotopy. If we arbitrarily assign an orientation to the non-trivial curves, the curves are either elements of the same homology class or cobound an annulus. Let $[\mathrm{m}]$ and $[l]$ represent the homology classes containing the oriented meridian and longitude respectively so that $[\mathrm{m}]$ and $[l]$ generate $H_{1}(T, \mathbb{Z})$. Recall that if $[\gamma]=a[m]+b[l]$ is a simple closed curve in $T$ then $a$ and $b$ are relatively prime.

Let the state $s_{i}$ contain $\gamma_{i}$, a simple closed curve that does not bound a disk. Then the homology class of $\left[\gamma_{i}\right]$ is given by the equation $\left[\gamma_{i}\right]=a_{i}[m]+b_{i}[l]$, where $a_{i}$ and $b_{i}$ are relatively prime. Let also the state $s_{j}$ contain $\gamma_{j}$, a simple 
closed curve that does not bound a disk such that $\left[\gamma_{j}\right]=a_{j}[m]+b_{j}[l]$. By hypothesis, $\left[\gamma_{i}\right] \bullet\left[\gamma_{j}\right] \neq 0$, implying that the curves $\gamma_{i}$ and $\gamma_{j}$ are not elements of the same cohomology class and the curves do not cobound an annulus. Using homology classes, we compute that

$$
\left[\gamma_{i}\right] \bullet\left[\gamma_{j}\right]=a_{i} b_{j}-b_{i} a_{j} .
$$

The cancellation curve $C$ is a simple closed curve that does not bound a disk in $T$. Let $[C]=g[m]+f[l]$ where $g$ and $f$ are relatively prime. We note that by Lemma 2.3. the curve $C$ does not intersect the curve $\gamma_{i}$ or the curve $\gamma_{j}$ since $C$ is a cancellation curve. We compute that

$$
\left[\gamma_{i}\right] \bullet[C]=a_{i} f-g b_{i}=0
$$

and

$$
\left[\gamma_{j}\right] \bullet[C]=a_{j} f-g b_{j}=0
$$

Note that

$$
0=a_{i} f-g b_{i}=a_{j} f-g b_{j}
$$

and so

$$
f\left(a_{i}-a_{j}\right)+g\left(b_{j}-b_{i}\right)=0 .
$$

We will consider the following three possibilities: $f \neq 0$ and $g \neq 0, f=0$, or $g=0$.

If we assume that $f=0$ then from 1 and 2 we obtain:

$$
-g b_{i}=0 \quad-g b_{j}=0
$$

and as a result $g=0$ or $b_{i}=b_{j}=0$. If $g=0$ then $C$ is not a cancellation curve because $C$ bounds a disk in the torus. If $b_{i}=b_{j}=0$ then this contradicts the fact that

$$
\left[\gamma_{i}\right] \bullet\left[\gamma_{j}\right] \neq 0
$$

Thus $f=0$ is not possible.

By the same argument, $g=0$ is not possible.

Suppose that $f \neq 0$ and $g \neq 0$. Recall that $g$ and $f$ are relatively prime, so that if $f \neq 0$ and $g \neq 0$ then either $f=1$ or $\frac{g}{f}$ is not an element of the integers. From 1 and 2 we obtain:

$$
a_{i}=g \frac{b_{i}}{f} \quad a_{j}=g \frac{b_{j}}{f}
$$

Note that $g, a_{i}$, and $a_{j}$ are integers and the pairs $(g, f),\left(a_{i}, b_{i}\right)$ and $\left(a_{j}, b_{j}\right)$ are relatively prime. This implies that $\frac{b_{i}}{f}$ and $\frac{b_{j}}{f}$ are integers. However, if $\frac{b_{i}}{f}$ 
is an integer $w$ such that $w \neq \pm 1$ then $a_{i}=g w$ and $b_{i}=f w$. This contradicts the fact that the pair $\left(a_{i}, b_{i}\right)$ was relatively prime. We obtain a similar result for the pair $\left(a_{j}, b_{j}\right)$. As a result, we determine that

$$
\pm 1=\frac{b_{i}}{f}=\frac{b_{j}}{f} \text {. }
$$

Hence, $b_{i}= \pm f$ and $b_{j}= \pm f$. Correspondingly, $a_{i}= \pm g$ and $a_{j}= \pm g$. This contradicts the fact that $\left[\gamma_{i}\right] \bullet\left[\gamma_{j}\right] \neq 0$.

Therefore, $C$ is not a cancellation curve for the torus $T$.

Let $F=T_{1} \sharp T_{2} \sharp \ldots \sharp T_{n}$. Let $\mathrm{C}$ be a cancellation curve for the surface $F$. Let $\left[m_{k}\right]$ and $\left[l_{k}\right]$ represent the homology classes containing the meridian and longitude of the torus $T_{k}$ respectively. Let $p_{*} H_{1}(F, \mathbb{Z}) \rightarrow H_{1}\left(T_{k}, \mathbb{Z}\right)$. We note that $p_{*}([C])=f\left[m_{k}\right]+g\left[l_{k}\right]$ with either $f \neq 0$ or $g \neq 0$ for some $T_{k}$. Otherwise $p_{*}[C]$ would bound a disk in each $T_{k}$. As a result, the curve $C$ divides the surface $F$ into two components, one of which contains the knot. If $C$ bounds a disk, then $C$ is not a cancellation curve. Hence $C$ bounds a component containing some states $s_{i}$ and $s_{j}$, contradicting the fact that $C$ is a cancellation curve.

Let $s_{i}$ and $s_{j}$ be states such that $p_{*}\left[s_{i}\right]$ contains a curve $\gamma_{i}$ and $p_{*}\left[s_{j}\right]$ contains a curve $\gamma_{j}$ such $\left[\gamma_{i}\right] \bullet\left[\gamma_{j}\right] \neq 0$. Let $\left[\gamma_{i}\right]=a_{i}\left[m_{k}\right]+b_{i}\left[l_{k}\right]$ and let $\left[\gamma_{j}\right]=a_{j}\left[m_{k}\right]+b_{j}\left[l_{k}\right]$ where the pairs $\left(a_{i}, b_{i}\right)$ and $\left(a_{j}, b_{j}\right)$ are relatively prime. Using the argument given previously, we eliminate the possibility that $f=0$ or $g=0$. We then consider the cases when $f \neq 0$ and $g \neq 0$. Using the same argument, we determine that

$$
b_{i}= \pm f \text { or } b_{j}= \pm f
$$

and combined with 3 this indicates that $a_{i}= \pm g$ and $a_{j}= \pm g$, contradicting our assumption that $\left[\gamma_{i}\right] \bullet\left[\gamma_{j}\right] \neq 0$. The cancellation curve $C$ was arbitrary and therefore $F$ has no cancellation curves.

Remark We note that the condition of theorem corresponds to the existence of two non-trivial, non-isotopic curves in each torus component projected from the states of the representation $(F, K)$.

\section{Virtual knot diagrams with one virtualized cross- ing}

Recall that a representation of a virtual knot diagram is minimal if no handles can be removed after a sequence of Reidemeister moves. In this section, we 
use the surface bracket polynomial to prove minimality for a class of virtual diagrams with one virtualized crossing. This enables us to show that many virtual knot diagrams are non-classical.

A classical crossing in a virtual knot diagram is virtualized by the following procedure: a tangle consisting of a single crossing is removed and replaced with a tangle consisting of the opposite crossing flanked by two virtual crossings. This procedure is illustrated in Figure 2 .

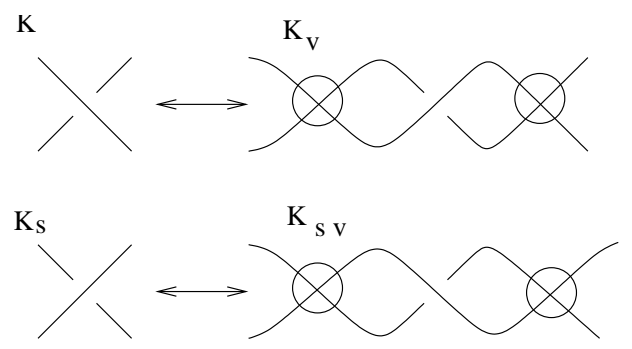

Figure 2: Virtualized Crossing

We consider the three knot diagrams as shown in Figure 3. The first diagram, labeled $K$ is a classical knot diagram formed by one isolated classical crossing $v$ and the classical tangle $T$. The second diagram, $K_{v}$ is obtained from $K$ by virtualizing the crossing $v$. The third diagram, $K_{s}$ is obtained by switching the isolated crossing.

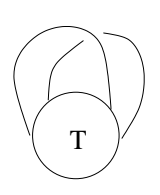

$\mathrm{K}$

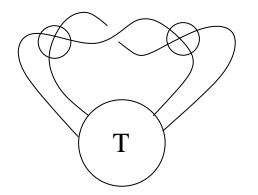

$\mathrm{K}_{\mathrm{V}}$

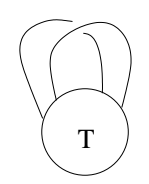

$\mathrm{K}_{\mathrm{S}}$

Figure 3: $K, K_{s}$ and the virtualized diagram: $K_{v}$

We apply the skein relation to the tangle $T$ and obtain the relation shown in Figure 4 where $\alpha$ and $\beta$ are coefficients in $\mathbb{Z}\left[A, A^{-1}\right]$.

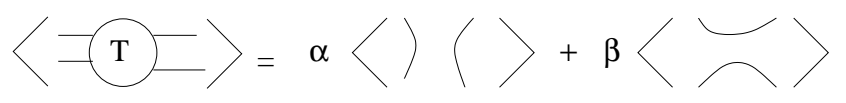

Figure 4: Skein Relation of Tangle T 
Applying the bracket skein relation and the relation shown in Figure 4, we determine that:

$$
\begin{aligned}
\langle K\rangle & =-A^{-3} \alpha-A^{3} \beta \\
\left\langle K_{s}\right\rangle & =-A^{3} \alpha-A^{-3} \beta .
\end{aligned}
$$

We note that $\left\langle K_{v}\right\rangle=\left\langle K_{s}\right\rangle$, see 9 . Hence

$$
\left\langle K_{v}\right\rangle=-A^{3} \alpha-A^{-3} \beta .
$$

In particular, if $K_{s}$ is an unknot and $w$ is the writhe of $K_{s}$. Then $\left\langle K_{s}\right\rangle=$ $(-A)^{-3 w}$ and $V_{K_{v}}(t)=V_{K_{s}}(t)=1$.

Lemma 3.1 Let $K$ be a classical knot or link, and let $K_{s}$ and $K_{v}$ be as in Figure 3. Let $\alpha$ and $\beta$ be defined as in Figure 4. Then

$$
\begin{aligned}
\langle K\rangle & =A^{-6}\left\langle K_{s}\right\rangle+\left(-A^{3}+A^{-9}\right) \beta \\
\alpha & =A^{-3}\left\langle K_{s}\right\rangle+A^{-6} \beta
\end{aligned}
$$

Proof Using the second part of equation 4.

$$
\left\langle K_{s}\right\rangle=-A^{3} \alpha-A^{-3} \beta \text {. }
$$

Solving for $\alpha$, we determine that:

$$
\alpha=-A^{-3}\left\langle K_{s}\right\rangle-A^{-6} \beta .
$$

Substitute into equation 4 and find:

$$
\langle K\rangle=A^{-6}\left\langle K_{s}\right\rangle+\left(-A^{3}+A^{-9}\right) \beta
$$

We introduce the following proposition.

Proposition 3.2 Let $K$ be a classical knot or link, and let $K_{s}$ and $K_{v}$ be as in Figure 3, Let $\alpha$ and $\beta$ be defined as in Figure 4, Then $\langle K\rangle=\left((-A)^{3}\right)^{ \pm 2}\left\langle K_{s}\right\rangle$ if and only if $\alpha=0$ or $\beta=0$.

Proof Suppose that $\langle K\rangle=\left((-A)^{3}\right)^{ \pm 2}\left\langle K_{s}\right\rangle$. We compute that:

$$
\begin{gathered}
\langle K\rangle=-A^{-3} \alpha-A^{3} \beta \\
\quad \text { and } \\
\left\langle K_{s}\right\rangle=-A^{3} \alpha-A^{-3} \beta
\end{gathered}
$$

where $\alpha$ and $\beta$ are non-zero elements of $\mathbb{Z}\left[A, A^{-1}\right]$ as shown in Figure 4 . As a result, we observe that:

$$
\langle K\rangle=-A^{-3} \alpha-A^{3} \beta
$$




$$
\begin{gathered}
\text { and } \\
A^{ \pm 6}\langle K\rangle=-A^{3} \alpha-A^{-3} \beta
\end{gathered}
$$

Now, taking +6 and -6 respectively, we find:

$$
\begin{gathered}
\langle K\rangle=-A^{-3} \alpha-A^{3} \beta \text { and } \\
\langle K\rangle=-A^{-3} \alpha-A^{-9} \beta \\
\text { or } \\
\langle K\rangle=-A^{-3} \alpha-A^{3} \beta \text { and } \\
\langle K\rangle=-A^{9} \alpha-A^{3} \beta
\end{gathered}
$$

These equations are contradictory unless either $\alpha=0$ or $\beta=0$. Suppose that $\alpha=0$. Using the skein relation,

$$
\begin{gathered}
\langle K\rangle=-A^{3} \beta \\
\text { and }\left\langle K_{s}\right\rangle=-A^{-3} \beta
\end{gathered}
$$

Therefore, $\langle K\rangle=A^{6}\left\langle K_{s}\right\rangle$. We may perform a similar computation if $\beta=0$ and determine that $\langle K\rangle=A^{-6}\left\langle K_{s}\right\rangle$.

Note that this proposition tells us that if $K_{s}$ is an unknot or an unlink then $K$ has the same bracket polynomial as an unknot or unlink if and only if $\alpha=0$ or $\beta=0$.

We consider a representation of the virtual knot diagram $K_{v}$ as a knot or a link embedded in a torus $F$ shown in Figure 5

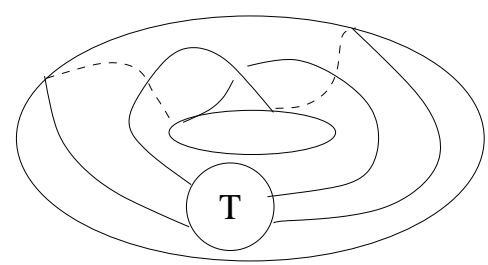

Figure 5: Representation: $\left(F, K_{v}\right)$

Theorem 3.3 Let $K$ be a classical knot or link diagram as in Figure 3 with associated links $K_{s}$ and $K_{v}$. If $\alpha$ and $\beta$, as determined in Figure 4, are both non-zero then $K_{v}$ is a non-classical and non-trivial virtual link.

Proof We obtain the two surface-state pairs $\left(F, K_{v+}\right)$ and $\left(F, K_{v-}\right)$ in Figure 6 from the skein relation. 

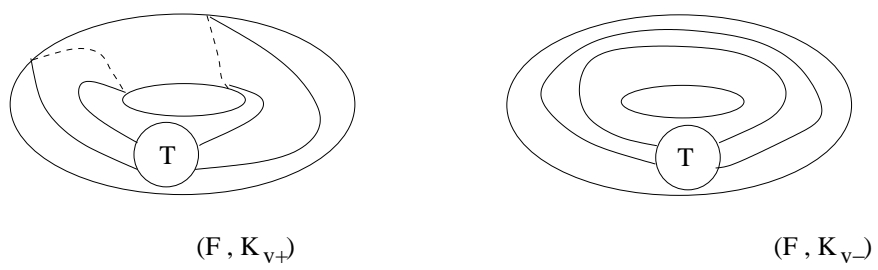

Figure 6: States in the Torus

Hence,

$$
\left\langle\left(F, K_{v}\right)\right\rangle=A^{-1}\left\langle\left(F, K_{v+}\right)\right\rangle+A\left\langle\left(F, K_{v-}\right)\right\rangle
$$

Combining this expansion with that states from [6 we obtain the relation shown in Figure 7.

$$
\langle(\mathrm{F}, \mathrm{K})\rangle=
$$
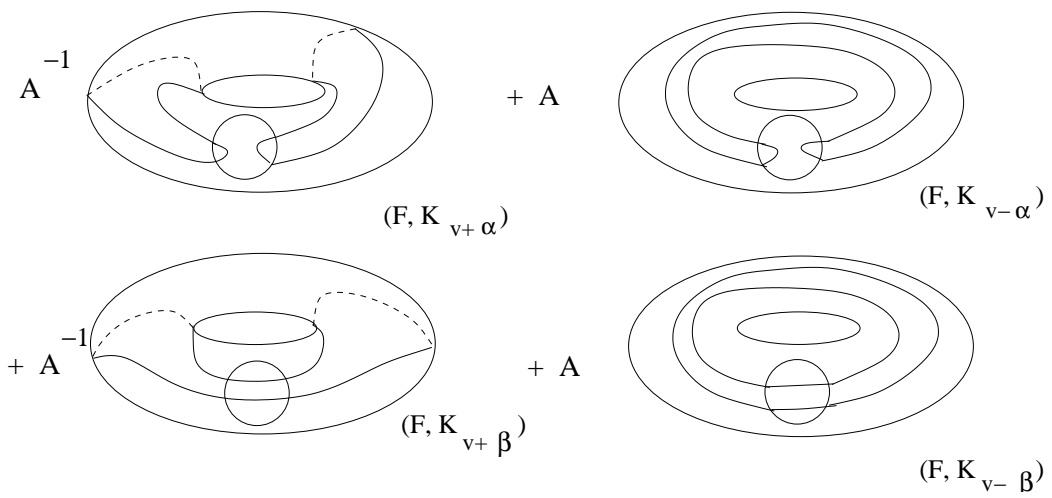

Figure 7: Surface-State Equation

We note that

$$
\begin{gathered}
\left\langle\left(F, K_{v}\right)\right\rangle=A^{-1}\left(\alpha\left\langle\left(F, K_{v+, \alpha}\right)\right\rangle+\beta\left\langle\left(F, K_{v+, \beta}\right)\right\rangle\right) \\
+A\left(\alpha\left\langle\left(F, K_{v-, \alpha}\right)\right\rangle+\beta\left\langle\left(F, K_{v-, \beta}\right)\right\rangle\right) .
\end{gathered}
$$

Referring to Figure [7] we observe that the states $\left(F, K_{v-, \alpha}\right)$ and $\left(F, K_{v+, \beta}\right)$ both contain a single curve that bounds a disk in $F$. As a result equation 5 reduces to

$$
\langle(F, K)\rangle=\left(A \alpha+A^{-1} \beta\right)+\left(A^{-1} \alpha\left\langle\left(F, K_{v+, \alpha}\right)\right\rangle+A \beta\left\langle\left(F, K_{v-, \beta}\right)\right\rangle .\right.
$$

Note that if both $\alpha$ and $\beta$ are non-zero, the subspace of curves generated by the surface-states spans the space of curves in the torus. 
We recall the following theorem from [23]:

Theorem 3.4 (V.F.R. Jones) If $K$ is a knot then $1-V_{K}(t)=W_{K}(t)(1-$ $t)\left(1-t^{3}\right)$ for some Laurent polynomial $W_{K}(t)$.

Note that if $V_{K}(t)$ is a monomial then $V_{K}(t)=1$. If $V_{K}(t)$ is a monomial, $\left(V_{K}(t)=t^{n}\right)$, then $1-t^{n}$ is divisible by $(1-t)$ and $\left(1-t^{3}\right)$ by Theorem 3.4. Hence, if $\mathrm{K}$ is a knot diagram and $\langle K\rangle=(-A)^{n}$ then $n=-3 w$, where $w$ is the writhe of $K$. We obtain the following corollary from this fact.

Corollary 3.5 If $K$ is a classical knot diagram with unknotting number one and non-unit Jones polynomial and $K_{s}$ is the unknot then $K_{v}$ is non-classical and non-trivial.

Proof Let $K$ have writhe $w$ then $K_{s}$ is the unknot with writhe $w \pm 2$. We obtain: $\left\langle K_{S}\right\rangle=(-A)^{-3(w \pm 2)}$. By Corollary $3.2 \alpha=0$ or $\beta=0$ if and only if $\langle K\rangle=(-A)^{-3 w}$. Since $\langle K\rangle \neq(-A)^{-3 w}$ then $\alpha \neq 0$ and $\beta \neq 0$. This indicates that the given representation of $K_{v}$ has no cancellation curves. The virtual genus of $K_{v}$ is one, indicating that $K_{v}$ is non-classical and non-trivial.

Remark Note that Corollary 3.5 does not eliminate the possibility that there exists a non-trivial classical knot diagram $K$ where both $K$ and $K_{s}$ have unit Jones polynomial, but $K_{v}$ is not detected by the surface bracket polynomial.

In [21], the following theorem is obtained from an analysis of the fundamental group.

Theorem 3.6 (Silver-Williams) Let $K$ be a non-trivial classical knot diagram, and $v$ is a classical crossing. If $K_{v}$ is the virtual knot diagram obtained by virtualizing $v$ in $K$ then $K_{v}$ is non-classical and non-trivial.

If $K$ is a non-trivial classical knot with $V_{K}(t)=1$ and $V_{K_{s}}(t)=1$ then the surface bracket polynomial would not detect $K_{v}$ even though the virtual genus is one via Theorem 3.6

We may generalize our procedure to demonstrate that a larger class of virtual knot diagrams is non-trivial and non-classical. Construct a virtual knot diagram from two classical tangles, $T$ and $S$ as shown in Figure 8 . The same arguments prove that if the tangles are expanded as shown in Figure 4 and the coefficients, $\alpha$ and $\beta$, are non-zero for both tangles then the virtual knot diagram has virtual genus one, whence the virtual knot diagram is non-classical and non-trivial. 


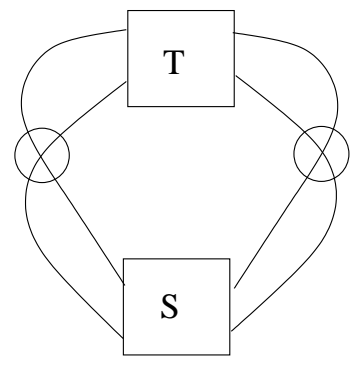

Figure 8: A virtual knot diagram constructed from two tangles

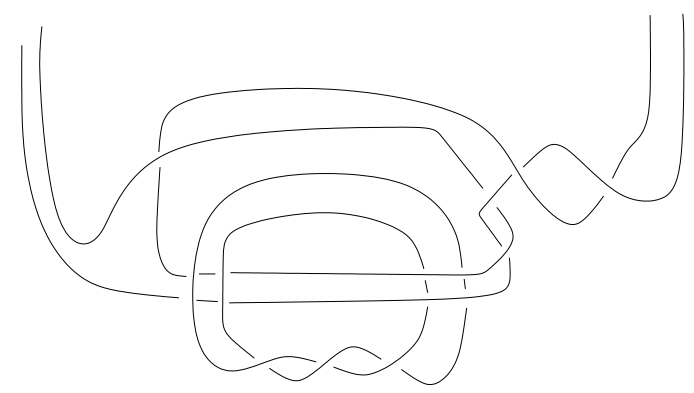

Figure 9: A tangle $T_{L}$ resulting in a link

\section{Undetectable Examples}

Given the tangle shown in Figure 9, we use the method given at the beginning of this section to construct a link diagram by taking a tangle sum with a single crossing. The link constructed from this tangle is shown in Figure 10. This link, $L$ and the corresponding link $L_{s}$ with a switched crossing have the property that both $L$ and $L_{s}$ have the same Jones polynomial as an unlink of two components. These link diagrams were constructed using the methods of [19].

For this link $L$, we note that $L_{v}$ is not detected by the surface bracket polynomial since by Corollary [3.2, $\alpha=0$. We thank Alexander Stoimenow for pointing out the usefulness of [19].

Remark We briefly comment on the case of a virtual knot diagram with a single virtual crossing and a classical tangle $T$. Let $K$ be such a virtual knot diagram. A schematic representation of this virtual knot diagram $(F, K)$ is shown in Figure [11 Let $[\mathrm{m}]$ and $[l]$ represent the meridian and longitude of the torus $F$. If we expand the tangle $\mathrm{T}$ as illustrated in Figure 4 we obtain:

$$
\langle K\rangle=\alpha+\beta
$$



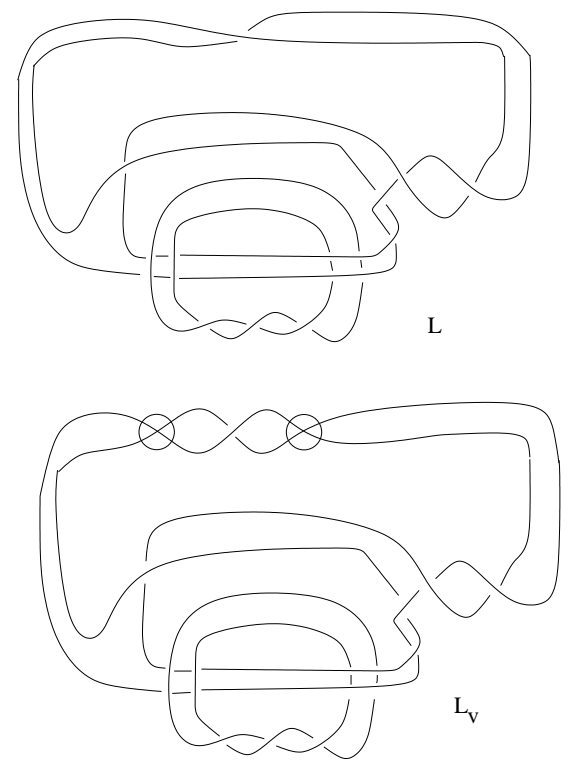

Figure 10: Link Diagrams: $L$ and $L_{v}$

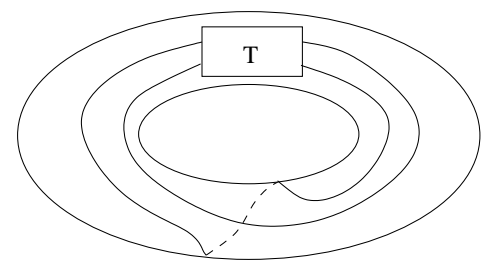

Figure 11: Representation of virtual knot diagram with 1 virtual crossing

$$
\langle(F, K)\rangle=\alpha\langle(F,[m])\rangle+\beta\langle(F,[m+2 l])\rangle
$$

Note that if $\alpha \neq 0$ and $\beta \neq 0$ then $K$ is non-classical and non-trivial. If $\alpha=0$ or $\beta=0$ then no decision can be made. In particular, we can construct a virtual link diagram with a single virtual crossing using the tangle shown in Figure 9. This link is not detected by the surface bracket polynomial.

\section{Other virtual knot diagrams}

We study other virtual knot diagrams and determine if these diagrams are non-classical and hence non-trivial using this technique. 
Kishino's knot is illustrated in Figure 12,

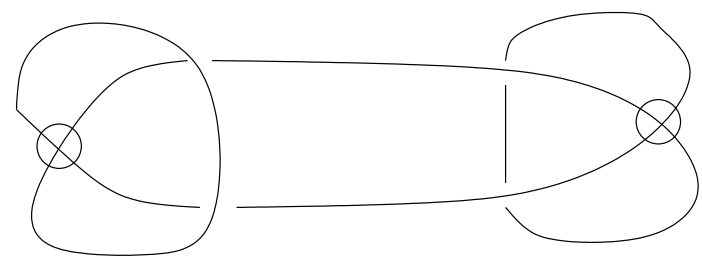

Figure 12: Kishino's Knot

This knot has a trivial fundamental group and bracket polynomial. This knot was determined to be non-trivial by the 3 -strand bracket polynomial 13 and the quaternionic biquandle [2]. Both of these methods involve intensive and difficult computation. The methods introduced in this paper demonstrate that Kishino's knot is non-classical and non-trivial and that the virtual genus of Kishino's knot is greater than zero. Recall the definition of virtual genus as given before Remark 2

Theorem 4.1 The virtual genus of Kishino's knot is two.

Corollary 4.2 Kishino's knot is non-trivial and non-classical.

Proof We show a genus two representation of Kishino's knot in Figure 13

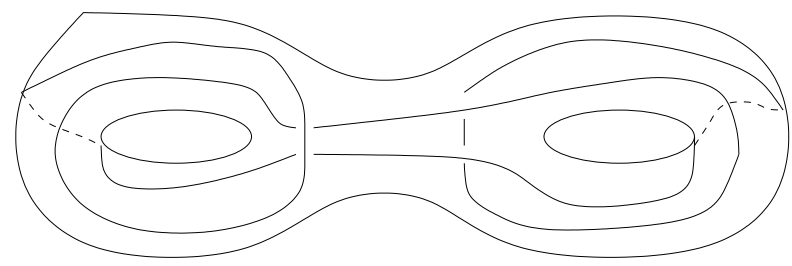

Figure 13: Genus Two Representation of Kishino's Knot

Note that Kishino's knot has 4 crossings. By application of the bracket polynomial, we obtain 16 surface-states from this representation.

We illustrate the 16 surface states in the following Figures.

We will denote state $\mathrm{i}$ as $s_{i}$ and the coefficients as $c_{i}$.

$$
\begin{array}{llll}
c_{1}=1 & c_{2}=A^{4} & c_{3}=A^{2} & c_{4}=A^{2} \\
c_{5}=A^{-2} & c_{6}=1 & c_{7}=1 & c_{8}=A^{2}
\end{array}
$$



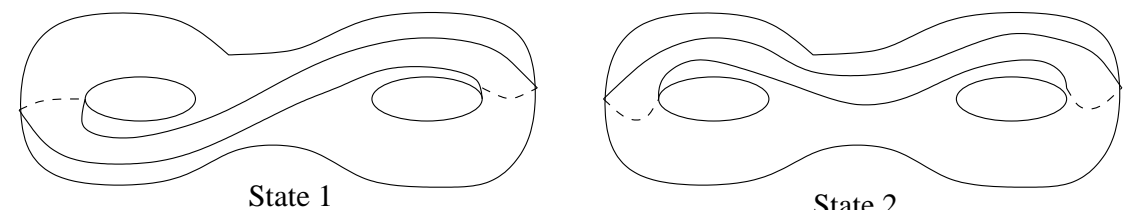

State 2

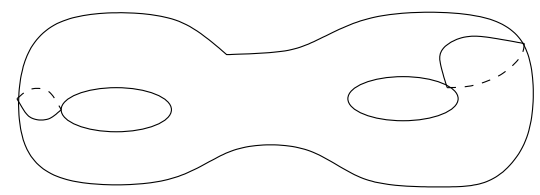

State 3

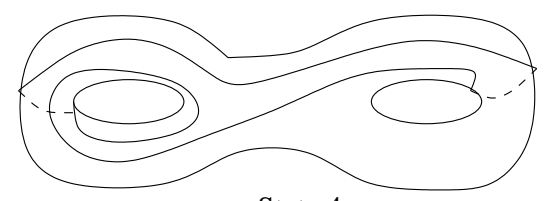

State 4

Figure 14: States of Kishino's Knot, 1-4

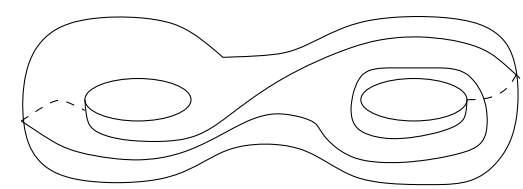

State 5

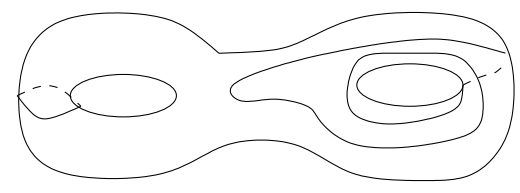

State 7

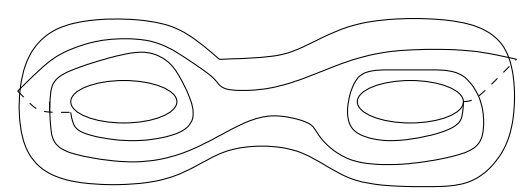

State 6

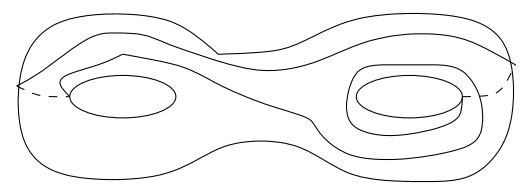

State 8

Figure 15: States of Kishino's Knot, 5-8

$$
\begin{aligned}
& c_{9}=A^{-2} \quad c_{10}=1 \quad c_{11}=1 \quad c_{12}=A^{2} \\
& c_{13}=A^{-4} \quad c_{14}=A^{-2} \quad c_{15}=A^{-2} \quad c_{16}=1
\end{aligned}
$$

We combine states with isotopy curves and obtain the following formula for the surface bracket polynomial.

$$
\begin{gathered}
\left(F, s_{1}\right)+A^{4}\left(F, s_{2}\right)+\left(A^{2}+A^{-2}\right)\left(F, s_{3}\right)+A^{2}\left(F, s_{4}\right) \\
+A^{-2}\left(F, s_{5}\right)+\left(F, s_{6}\right)+\left(F, s_{7}\right)+A^{2}\left(F, s_{8}\right) \\
+\left(F, s_{10}\right)+A^{-4}\left(F, s_{13}\right)+A^{-2}\left(F, s_{14}\right)+\left(F, s_{16}\right)
\end{gathered}
$$

Note that the states $s_{3}, s_{10}$, and $s_{14}$ modulo 2 span the entire space of homology classes of curves in the connected sum of two tori. The fact that these curves span the homology group is invariant under isotopy of the knot in the surface and invariant under homeomorphisms of the surface. Hence, Kishino's knot is 


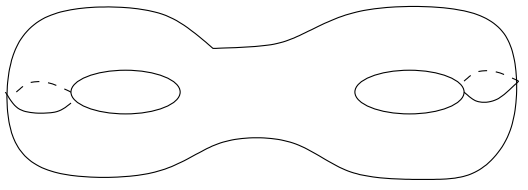

State 9

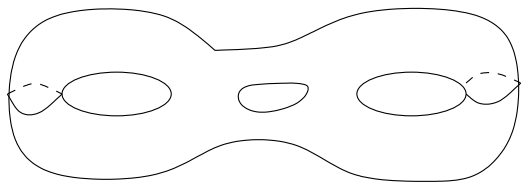

State 11

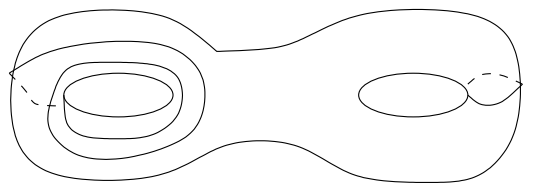

State 10

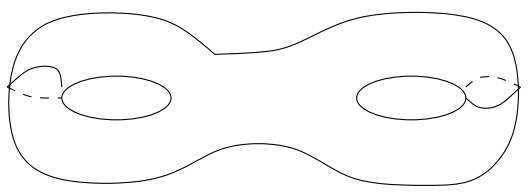

State 12

Figure 16: States of Kishino's Knot, 9-12

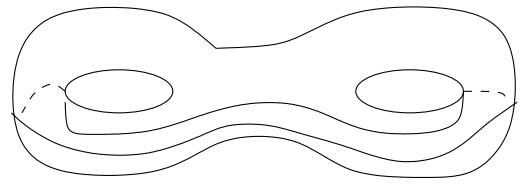

State 13

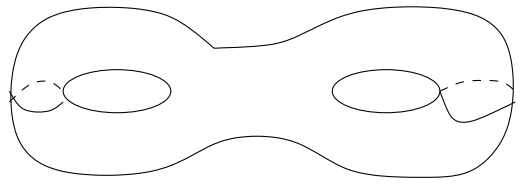

State 15

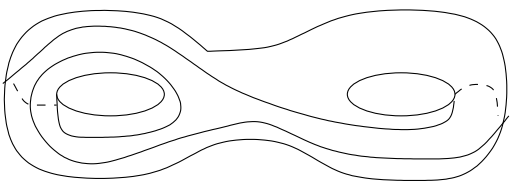

State 14

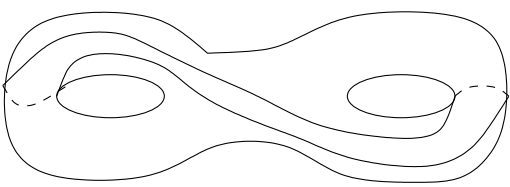

State 16

Figure 17: States of Kishino's Knot, 12-16

not equivalent to a knot that admits a cancellation curve. Therefore, the virtual genus of Kishino's knot is two.

We consider a slight modification of Kishino's knot, as illustrated in Figure 18 .

This virtual knot diagram is undetected by the fundamental group and the 1strand and 2-strand bracket polynomial [4]. The knot has 6 classical crossings, and expands into 32 states. We may represent this virtual knot diagram as a knot diagram on the connected sum of two tori, and compute the expanded states and coefficients in each torus. This process forces us to conclude that there are no cancellation curves in this surface. As a result we obtain:

Proposition 4.3 The modified Kishino's knot is non-trivial and non-classical. 


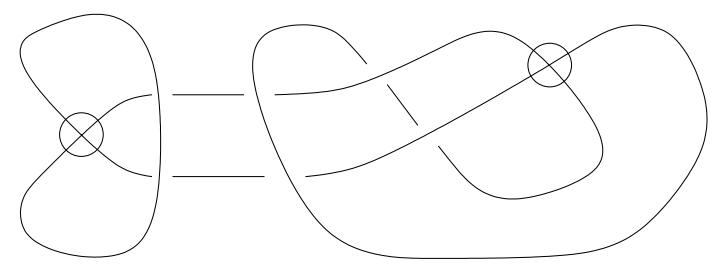

Figure 18: Modified Kishino's Knot

Proof Use the method given in the previous proof. Compute the surface bracket polynomial and compare the rank of the equivalence classes of curves in states with non-zero coefficients.

We consider a further modification of this virtual knot diagram, as shown in Figure 19.

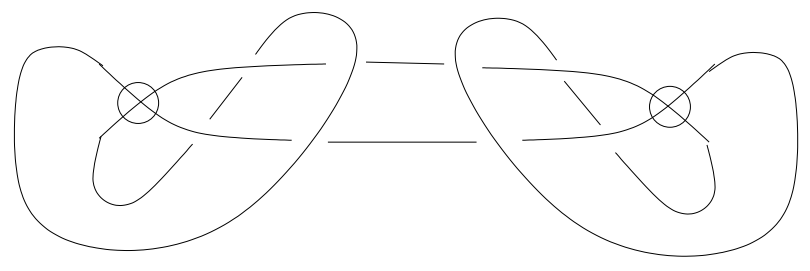

Figure 19: New Knot

Theorem 4.4 The virtual knot diagram shown in Figure 19 is non-trivial and non-classical.

In fact, this diagram is part of an infinite class of knots that is not detected by the bracket polynomial. We will prove that the members of the infinite class are detected by the surface bracket polynomial. This includes the case of Theorem 4.4 .

Theorem 4.5 There is an infinite family of non-trivial virtual knot diagrams obtained by modifying Kishino's knot. These virtual knot diagrams are not detected by the bracket polynomial but are detected by the surface bracket polynomial. A schematic diagram of this family is shown in Figure 20.

Proof We denote the members of this family as $P_{n}$, where $n$ denotes the number of inserted twists. As a result, $P_{0}$ refers to the diagram shown in Figure 19. By applying the surface bracket polynomial to the knot shown in Figure 19. we obtain the following states with non-zero coefficients. These states are 


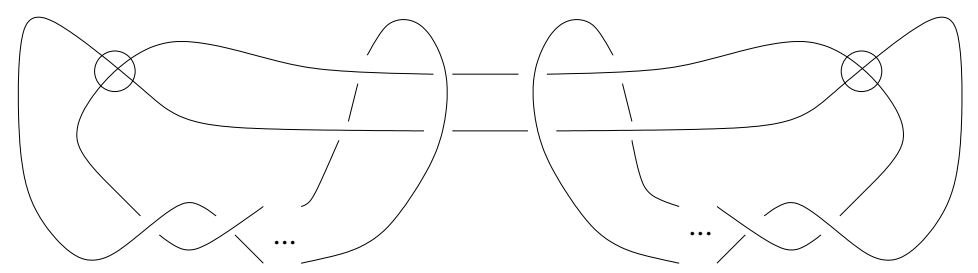

Figure 20: Schematic of the Family
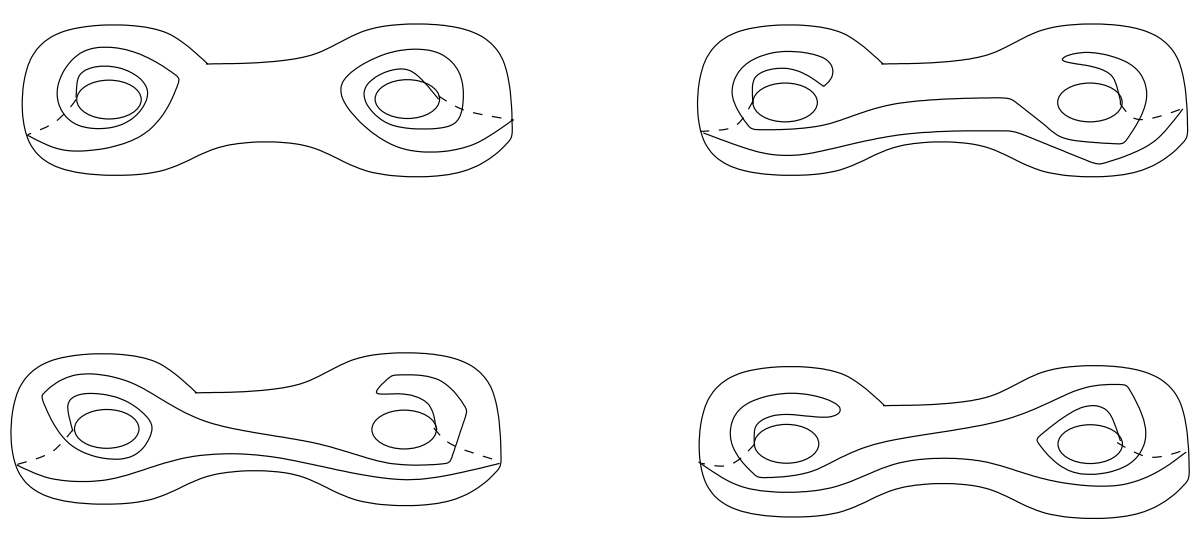

Figure 21: States of the New Knot shown in Figure 19

sufficient to ensure that no cancellation curves exist in the surface. Hence, the virtual genus of this diagram is two. We expand the diagram $P_{n}$ to obtain the state sum illustrated in Figure 22 using the skein. A lengthy calculation shows that the coefficients $c_{1}, c_{2}, c_{3}$ and $c_{4}$ are non-zero. The states shown in Figure 21] are obtained by expanding the state with coefficient $c_{1}$ from Figure 22. The expansion of the states coefficients $c_{2}, c_{3}$ and $c_{4}$ does not involve these states. Consequently, the states shown in Figure 21 are not cancelled and have nonzero coefficients in the final state sum. These states are sufficient to ensure that no cancellation curves exist. Hence, these virtual knot diagrams have virtual genus two. As a result, they are non-trivial and non-classical.

The knot diagram in 19 is not detected by the 1 and 2-strand bracket polynomial, but it is detected by the 3-strand bracket polynomial. However it is simpler to apply to bracket polynomial a genus 2 representation of this knot. We do not know if the other members of the infinite family are detected by the 3 -strand bracket polynomial. These computations are extremely complex, and we are currently unable to complete the calculations on a computer.

For a virtual knot diagram with $n$ classical crossings, the 3 -strand bracket 


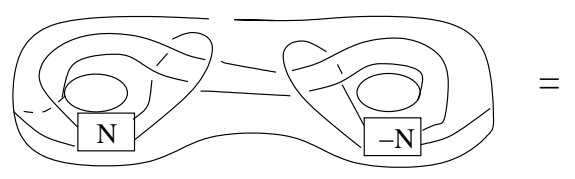

$\mathrm{c}_{1}$
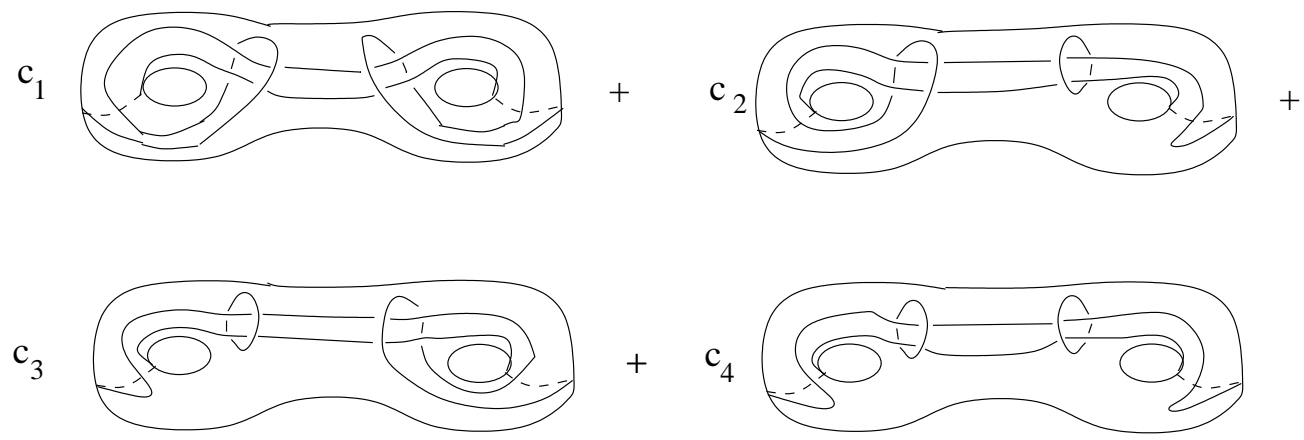

Figure 22: Partial State Expansion

polynomial has complexity of order $2^{9 n}$. These computations are considered in depth in [4.

Remark We conjecture that the 3-strand bracket polynomial detects virtual knot diagrams. The states of the 3-strand bracket polynomial may reflect the geometry of the minimal surface.

Remark Kodakami's work on the detection of virtual knot diagrams is closely related to this approach [14. We note that his approach works for diagrams that are non-trivial in the flat category. The flat versions of the virtual knot diagrams in Figure 20 and in Figure 18 are trivial, indicating that Kodakami's method would not detect these knots.

\section{Virtual knot diagrams with two virtualized cross- ings}

We conclude this paper by considering the following class of virtual knot diagrams. Let $K$ be a classical knot diagram, consisting of a classical 4-4 tangle $T$, occuring in an annulus, and two isolated crossings. The isolated crossings as 
chosen so the knot $K_{s}$ with the isolated crossings switched in the unknot. Let $K_{v}$ denote the modified diagram produced by virtualizing the isolated crossings. These Figures are illustrated in Figure 23]. Note that the genus of the characterization of $K_{v}$ is bounded above by genus 2 .

We apply our new method to a virtual knot diagram constructed by applying two virtualizations. Observe that in some cases it is possible to determine that a virtual knot diagram is non-classical and non-trivial without a full expansion of the bracket polynomial.

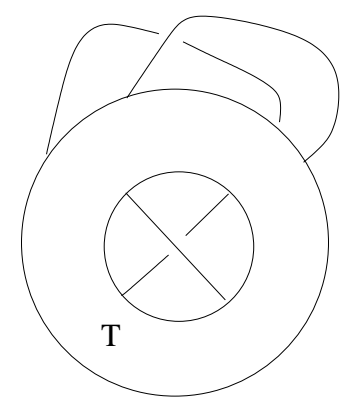

K

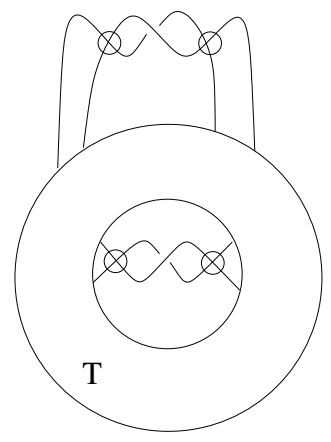

$\mathrm{K}_{\mathrm{v}}$

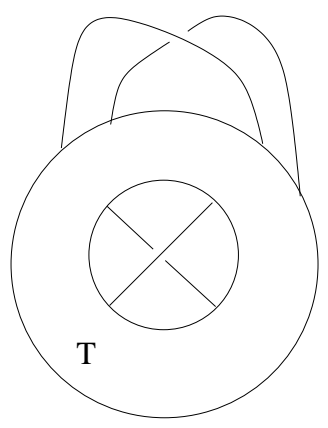

$\mathrm{K}_{\mathrm{S}}$

Figure 23: $K, K_{v}$, and $K_{s}$

The equivalence classes of states that arise from application of the bracket polynomial to 4-4 tangle in an annulus have not been determined. As a result we restrict our attention to a modification of $K$. The knot $K^{\prime}$ is obtained by applying a sequence of Reidemeister moves to the classical knot diagram $K$. The diagram $K^{\prime}$ consists of a classical 4-4 tangle $T^{\prime}$, contained in a disk, and two isolated crossings. We construct $K_{v}^{\prime}$ and $K_{s}^{\prime}$ as before. The diagrams $K^{\prime}$, $K_{v}^{\prime}$ and $K_{s}^{\prime}$ are illustrated in Figure 24,

The diagrams $K$ and $K^{\prime}$ are equivalent, but two virtual diagrams $K_{v}$ and $K_{v}^{\prime}$ are not necessarily virtually equivalent. The diagrams $K_{s}$ and $K_{s}^{\prime}$ are both unknots. We consider the bracket polynomial:

$$
\begin{aligned}
\langle K\rangle & =(-A)^{3 n}\left\langle K^{\prime}\right\rangle \\
\left\langle K_{s}\right\rangle & =(-A)^{3 n}\left\langle K_{s}^{\prime}\right\rangle \\
\left\langle K_{s}\right\rangle & =\left\langle K_{v}\right\rangle \\
\left\langle K_{s}^{\prime}\right\rangle & =\left\langle K_{v}^{\prime}\right\rangle
\end{aligned}
$$

where $n$ reflects the number of Reidemeister I moves. 


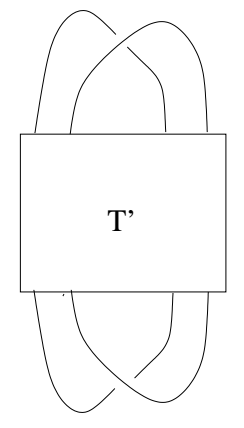

$\mathrm{K}^{\prime}$

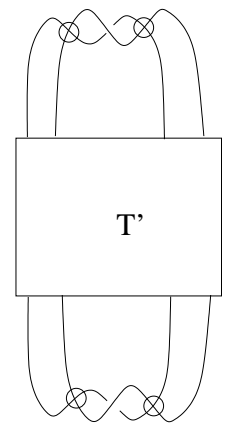

$\mathrm{K}_{\mathrm{V}}$

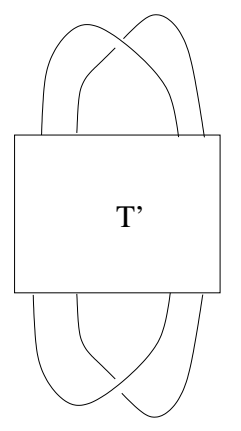

K's

Figure 24: $K^{\prime}, K_{v}^{\prime}$, and $K_{s}^{\prime}$

Expanding the tangle $T^{\prime}$ using the skein relation, we obtain a linear combination of the twelve elements of the $4^{\text {th }}$ Temperly-Lieb algebra [10] with coefficients in $\mathbb{Z}\left[A, A^{-1}\right]$. The twelve elements of the $4^{t h}$ Temperly-Lieb algebra are shown in Figure 25.

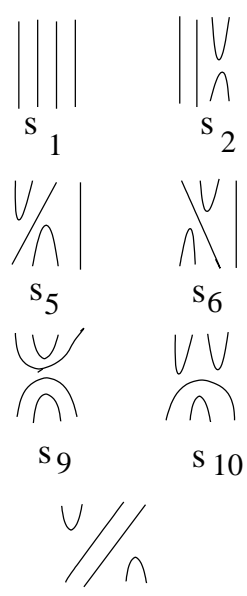

$\mathrm{s}_{13}$
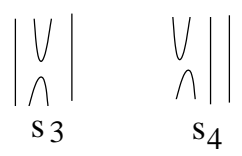

V/ $/ /$

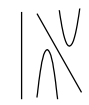

$\mathrm{s}_{7} \quad \mathrm{~s}_{8}$

(U) $\quad V$

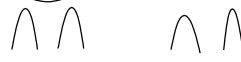

$\mathrm{s}_{11} \mathrm{~s}_{12}$

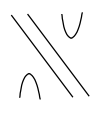

$\mathrm{s}_{14}$

Figure 25: Generators of the $4^{\text {th }}$ Temperly-Lieb Algebra

We will refer to the labels assigned to each state later in this section. We consider a representation of $K_{v}^{\prime}$ in the connected sum of two tori. Applying the skein relation to the isolated crossings, we obtain an equation with four states. This equation is illustrated in Figure 26]

For some virtual knot diagrams it is possible to determine (or bound) the virtual 


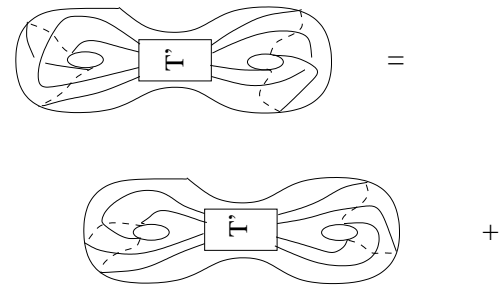

State AA

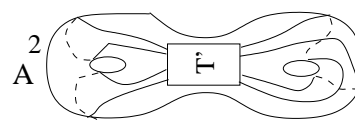

State BA

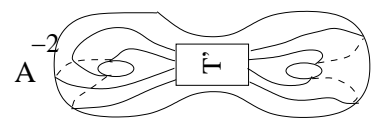

State AB

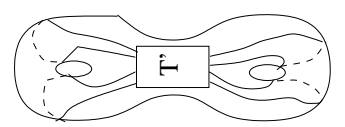

State BB

Figure 26: Expansion of a Representation of $K_{v}^{\prime}$

genus of the representation without a full expansion of $T^{\prime}$.

We introduce an example constructed from a classical knot diagram with unknotting number two. Consider the classical knot diagram $K^{\prime}$ with unknotting number 2, shown in Figure 27. The diagram $K$ has an associated virtualized diagram $K_{v}^{\prime}$ constructed as above.

Theorem 5.1 The virtual knot diagram $K_{v}^{\prime}$ has virtual genus two.

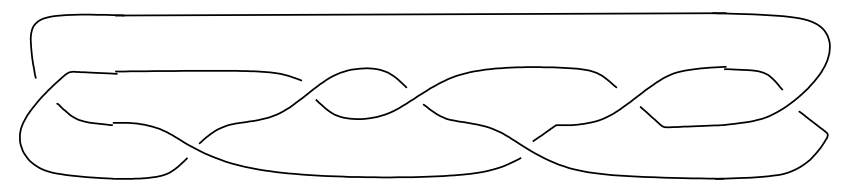

Figure 27: Knot $K$ with Unknotting Number 2

Proof We decompose $K$ into two isolated crossings and a classical 4-4 tangle $T^{\prime}$, illustrated in Figure 28

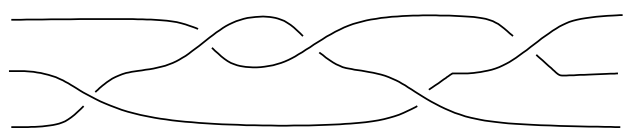

Figure 28: The tangle $T^{\prime}$ 

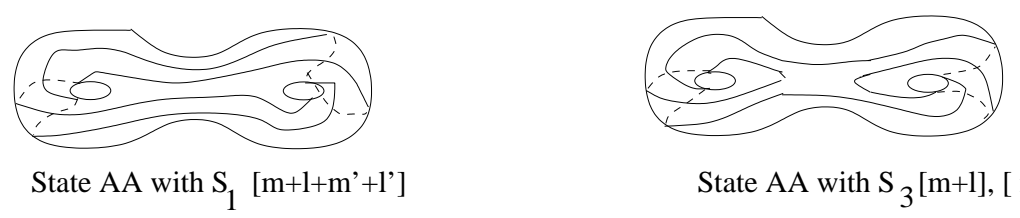

State AA with $S_{3}[\mathrm{~m}+1],\left[\mathrm{m}^{\prime}+\mathrm{l}^{\prime}\right],\left[\mathrm{m}+1+\mathrm{m}^{\prime}+\mathrm{l}^{\prime}\right]$

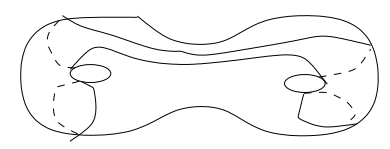

State BB with $S_{2}[\mathrm{~m}],\left[\mathrm{m}^{\prime}\right],\left[\mathrm{m}+\mathrm{m}^{\prime}\right]$

Figure 29: Non-Zero States

In Figure 25 we list the states obtained from a bracket expansion of a $4-4$ tangle. These states correspond to the generators of the $4^{t h}$ Temperly-Lieb algebra. The states and coefficients obtained from the bracket expansion of $T^{\prime}$ are:

$$
\begin{gathered}
A^{-1} s_{1}+\left(A^{9}-2 A^{5}+2 A\right) s_{3}+\left(-A+2 A^{-3}-A^{-7}\right) s_{4} \\
+\left(A^{7}-2 A^{3}+2 A^{-1}-A^{-5}\right) s_{5}+\left(-A^{3}+A^{-1}\right) s_{6}
\end{gathered}
$$

Inserting this expansion into the relation obtained from the skein relation, as shown in Figure 26] we have the following non-zero states shown in Figure 29.

These states are sufficient to prevent the presence of any cancellation curves.

Acknowledgments The views expressed herein are those of the authors and do not purport to reflect the position of the United States Military Academy, the Department of the Army, or the Department of Defense. Much of this effort was sponsored for the second author by the Defense Advanced Research Projects Agency (DARPA) and Air Force Research Laboratory, Air Force Materiel Command, USAF, under agreement F30602-01-2-05022. The U.S. Government is authorized to reproduce and distribute reprints for Government purposes notwithstanding any copyright annotations thereon. The views and conclusions contained herein are those of the authors and should not be interpreted as necessarily representing the official policies or endorsements, either expressed or implied, of the Defense Advanced Research Projects Agency, the Air Force Research Laboratory, or the U.S. Government. It gives the second author great pleasure to acknowledge support from NSF Grant DMS-0245588. (Copyright 2005.) 


\section{References}

[1] Glen E. Bredon, Topology and Geometry, Graduate Texts in Mathematics, Springer-Verlag, 1997 MathReview

[2] Andrew Bartholomew and Roger Fenn, Quaternionic Invariants of Virtual Knots and Links, Preprint

[3] J. Scott Carter, Seiichi Kamada, and Masahico Saito, Stable equivalence of knots on surfaces and virtual knot cobordisms, Knots 2000 Korea, Vol. 1 (Yongpyong), Journal of Knot Theory and its Ramifications Vol. 11, No. 3, p. 311-332, 2002 MathReview

[4] H.A. Dye, Virtual Knots undetected by 1 and 2-strand Bracket Polynomials, Preprint, arXiv:math.GT/0402308

[5] Roger Fenn, Colin Rourke, Brian Sanderson, The rack space, Trans. Amer. Math. Soc. (to appear) arXiv:math.GT/0304228

[6] Morris W. Hirsch, Differential Topology, Springer-Verlag, Graduate Texts in Mathematics, 1997

[7] Naoko Kamada and Seiichi Kamada, Abstract link diagrams and virtual Knots, Journal of Knot Theory and its Ramifications, Vol. 9 No. 1, p. 93-109, World Sci. Publishing, 2000 MathReview

[8] Louis H. Kauffman, Detecting Virtual Knots, Atti del Seminario Matematico e Fisico dell'Universite di Modena, Vol. 49, suppl., p. 241-282, Univ. Modena, 2001 MathReview

[9] Louis H. Kauffman, Virtual Knot Theory, European Journal of Combinatorics, Vol. 20, No. 7, p. 663-690, Academic Press, 1999 MathReview

[10] Louis H. Kauffman and Sostenes L. Lins, Temperly-Lieb Recoupling Theory and Invariants of 3-Manifolds, Annals of Mathematics Studies, Princeton University Press, 1994 MathReview

[11] Louis H. Kauffman and Vassily Olegovich Manturov, Virtual Biquandles, Preprint, arXiv:math.GT/0411243

[12] Louis H. Kauffman and Vassily Olegovich Manturov, Virtual knots and links, (to appear in proceedings of the Keldysh conference held in 2004 and to be expanded into a book "Virtual Knot Theory") arXiv:math.GT/0502014

[13] Toshimasa Kishino and Shin Satoh, A note on classical knot polynomials, Journal of Knot Theory and its Ramifications, Vol 13, No. 7, p. 845-856, 2004 MathReview

[14] Teruhisa Kadokami, Detecting non-triviality of virtual links, Journal of Knot Theory and its Ramifications, Vol. 12, No. 6, p. 781-803, 2003 MathReview

[15] Greg Kuperberg, What is a Virtual Link? Algebraic and Geometric Topology, Vol. 3, No. 20, p. 587-591, 2003 MathReview 
[16] Greg Kuperberg, private conversation

[17] Vassily O. Manturov, Kauffman-Like Polynomial and Curves in 2-Surfaces Journal of Knot Theory and its Ramifications, Vol. 12, No. 8, p. 1131-1144, 2003 MathReview

[18] Sergei Matveev, Algorithmic Topology and Classification of 3-Manifolds, Springer-Verlag, 2003 MathReview

[19] Shalom Eliahou, Louis H. Kauffman, Morwen Thistlethwaite it Infinite families of links with trivial Jones polynomial, Topology, Vol. 42, No. 1, p. 155169 (2003) MathReview

[20] V.V. Paraslov and A.B. Sossinky, Knots, Links, Braids and 3-Manifolds; An Introduction to New Invariants in Low-Dimensional Topology, American Mathematical Society, Translations of Mathematical Monographs, 1996 MathReview

[21] Daniel Silver and Susan Williams, On a Class of Virtual Knots with Unit Jones Polynomial, Journal of Knot Theory and its Ramifications, Vol. 13, No. 3, p. 367-371, 2004 MathReview

[22] Vaughan F.R. Jones, The annular structure of subfactors, Essays on geometry and related topics, Vol. 1, 2, No. 38 of Monogr. Enseign. Math., p. 401-463, Enseignement Math., Geneva, 2001. MathReview

[23] Vaughan F.R. Jones, Hecke Algebra Representations of Braid Groups and Link Polynomials, The Annals of Mathematics, second Series, Vol. 126, Issue 2 (Sep., 1987), p. 335-388 MathReview

MADN-MATH, United States Military Academy

646 Swift Road, West Point, NY 10996, USA

and

Department of Mathematics, Statistics and Computer Science

University of Illinois at Chicago, 851 South Morgan St

Chicago, IL 60607-7045, USA

Email: hdye@ttocs.org, kauffman@uic.edu

Received: 31 May 2004 